\title{
Percutaneous Endoscopic Gastrostomy Tube Dislocation 2 Days after Insertion with Consequent Peritonitis
}

\author{
Janež J* \\ Department of Abdominal Surgery, University Medical Centre Ljubljana, Slovenia
}

*Corresponding author: Jurij Janež, Department of Abdominal Surgery, University Medical Centre Ljubljana, Zaloška Cesta 7, 1525 Ljubljana, Slovenia, Tel: +38651315815; Email: jurij.janez@gmail.com

\section{Case Report \\ Volume 2 Issue 3}

Received Date: April 22, 2018

Published Date: May 16, 2018

DOI: $10.23880 / \mathrm{mjccs}-16000151$

\section{Abstract \\ Percutaneous endoscopic gastrostomy is a procedure that involves an endoscopic guided insertion of gastrostomy tube for purposes of enteral feeding. It is usually performed in patients after brain stroke or patients with malignant disease of throat that are unable of swallowing. In some cases, the gastrosotmy tube can become dislocated, allowing the gastric content to escape into the abdominal cavity, causing intra-abdominal abscess or peritonitis. This paper presented a case of a-80-year old male patient, who needed emergency operation due to displaced gastrostomy tube 2 days after insertion.}

Keywords: Percutaneous Endoscopic Gastrostomy; Tube Displacement; Emergency Surgery; Haemorrhage; Jejunostomy

Abbreviations: PEG: Percutaneous Endoscopic Gastrostomy; CT: Computed Tomography.

\section{Introduction}

Percutaneous endoscopic gastrostomy (PEG) is a procedure often needed in patients after brain stroke or with throat cancer that are unable of normal enteral feeding. The gastrostomy tube is inserted under endoscopic guidance. This technique was first described by Gauderer in 1980. Different variations of the technique include the pull (Ponsky), push (Sachs-Vine), introducer (Russell), and Versa (T-fastener) methods [1].

Patients who are unable to move food from their mouth to their stomach are the ones who commonly need PEG tube placement. This includes those with neurologic disorders such as stroke, cerebral palsy, brain injury, amyotrophic lateral sclerosis, and impaired swallowing
[2]. In addition, patients who have trauma, cancer, or recent surgery of the upper gastrointestinal tract the respiratory tract may require this procedure to maintain nutritional intake. Gut decompression may be needed in patients who have abdominal malignancies causing gastric outlet or small-bowel obstruction or ileus [3]. This paper presented a case of an 80-year-old male patient, who needed emergency operation 2 days after PEG insertion due to PEG displacement and consequent peritonitis.

\section{Case Report}

80-year-old male patient, after intracranial haemorrhage, had PEG insertion for purposes of enteral feeding. Two days after the procedure his abdomen became tender and very painful on palpation. The staff nurses noticed, that the PEG tube is displaced and that there is secretion around gastrostomy tube. Abdominal $\mathrm{x}$ - 


\section{Medical Journal of Clinical Trials \& Case Studies}

ray was performed, that showed free intraperitoneal air under the right diaphragm (Figure 1). Abdominal computed tomography (CT) was also performed, that showed a large amount of free intraperitoneal air and the inner cuff of the PEG, that seemed to be dislodged outside the gastric lumen under the abdominal wall (Figure 2). We decided for an emergency surgery. Exploratory laparotomy was performed. There was a free intraperitoneal fluid and signs of peritonitis. We took a fluid sample for microbiology. There was a leakage of gastric content around the PEG, which was displaced. PEG was removed, gastric wall sutured and irrigation of the abdominal cavity was performed. A feeding jejunostomy tube was inserted in to the proximal jejunum. Abdominal drain was inserted and the abdominal wall was closed with interrupted resorbable sutures. Skin wound was closed with staples. The patient was transferred to the intensive care unit.

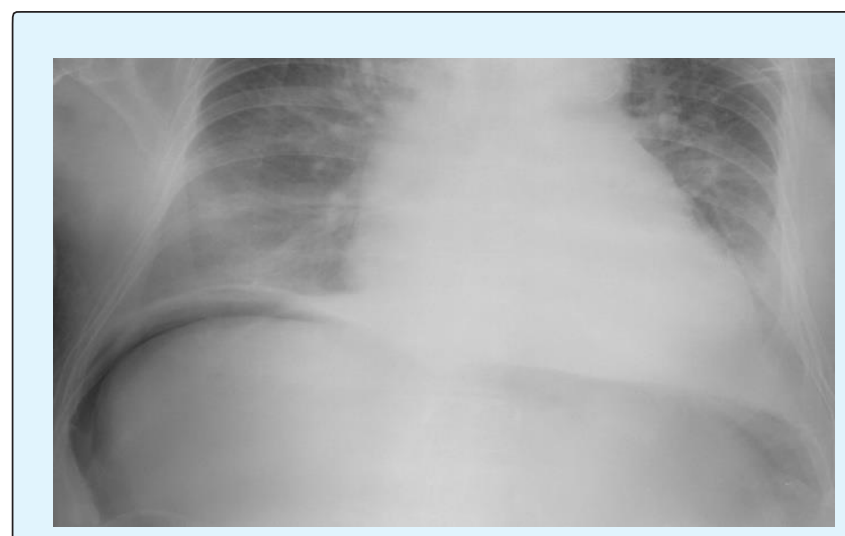

Figure 1: Abdominal and chest $\mathrm{x}$-ray, showing free intraperitoneal air under the right diaphragm.

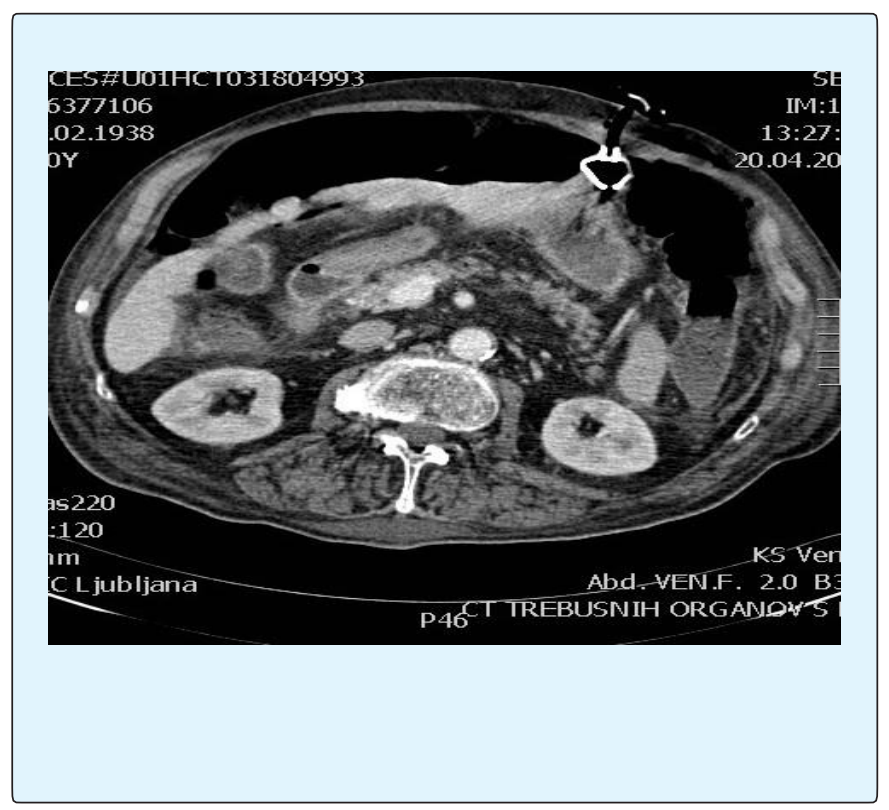

Figure 2: Abdominal CT, showing free intraperitoneal air and inner cuff of the PEG that is partially outside the gastric lumen.

\section{Discussion}

Some adults and children are unable to swallow or eat and drink enough. Insertion of a percutaneous endoscopic gastrostomy (PEG) tube may enable long-term feeding, fluid and/or medication administration. The procedure involves gastroscopy under sedation to identify tube placement site, place the tube and check it has been placed correctly. Serious complications include peritonitis and perforation of the colon. Frequent observations immediately after placement are essential. Complications can occur with the PEG placement, such as pain at the PEG site, leakage of stomach content around the tube site, and dislodgment or malfunction of the tube. Possible complications also include infection of the PEG site, aspiration (inhalation of gastric content into the lungs), bleeding and perforation (an unwanted hole in the bowel wall) [4]. With good nursing care, complications can be avoided or dealt with promptly [5]. PEG feeding will reduce morbidity and mortality in many such patients by reversing malnutrition. The increasing numbers of elderly patients with chronic diseases have resulted in an increased demand for PEG placement that has stretched resources. Many patients who are referred for PEGs are frail and the procedure is associated with complications. Careful management and support for the carriers in the community are essential. Not all patients benefit from PEG feeding [6].

\section{Conclusion}

Feeding through a PEG tube is the desirable method for patients with dysphagia or who are unable to feed orally. Careful management of patients with PEG should be employed, since serious complications can occur.

\section{References}

1. Gauderer MW, Ponsky JL, Izant RJ Jr (1980) Gastrostomy without laparotomy: a percutaneous endoscopic technique. J Pediatr Surg 15(6): 872875.

2. Kak M, Issa NP, Roos RP, Sweitzer BJ, Gottlieb O, et al. (2017) Gastrostomy tube placement is safe in advanced amyotrophic lateral sclerosis. Neurol Res 39(1):16-22.

3. Gomes CA Jr, Andriolo RB, Bennett C, Lustosa SA, Matos D, et al. (2015) Percutaneous endoscopic 


\section{Medical Journal of Clinical Trials \& Case Studies}

gastrostomy versus nasogastric tube feeding for adults with swallowing disturbances. Cochrane Data base Syst Rev CD008096.

4. Friginal-Ruiz AB, Lucendo AJ (2015) Percutaneous Endoscopic Gastrostomy: A Practical Overview on Its Indications, Placement Conditions, Management, and Nursing Care. Gastroenterol Nurs 38(5): 354366.

5. Haywood S (2012) PEG feeding tube placement and aftercare. Nurs Times 108(42): 20-22.

6. Pennington C (2002) To PEG or not to PEG. Clin Med (Lond) 2(3): 250-255. 